\title{
Towards Understanding of Relationship among Pareto Optimal Solutions in Multi-dimensional Space via Interactive System
}

\author{
Keiki Takadama ${ }^{1}$, Yuya Sawadaishi ${ }^{1}$, Tomohiro Harada ${ }^{1}$, Yoshihiro Ichikawa ${ }^{1}$, \\ Keiji Sato ${ }^{1}$, Kiyohiko Hattori ${ }^{1}$, Hiroyoki Sato ${ }^{1}$, and Tomohiro Yamaguchi ${ }^{2}$ \\ ${ }^{1}$ The University of Electro-Communications \\ 1-5-1 Chofugaoka, Chofu, Tokyo,182-8558 Japan \\ \{keiki, hattori, sato\}@hc.uec.ac.jp, \\ \{sawadaishi, harada,yio,keiji\}@cas.hc.uec.ac.jp \\ ${ }^{2}$ Nara National College of Technology \\ 22, Yata-cho, Yamatokoriyama, Nara 639-1080 Japan \\ yamagucheinfo.nara-k.ac.jp
}

\begin{abstract}
This paper proposes the interactive system that can help humans to understand the trade-off relationship of Pareto optimal solutions (e.g., good products from a certain aspect) in multi-dimensional space. For this purpose, the following two methods are proposed from the viewpoint of the number of evaluation criteria which should be considered by a user at one time: (i) the two fixed evaluation criteria are employed to evaluate the solutions; and (ii) some evaluation criteria selected by a user (i.e., the number of the evaluation criteria is varied by a user) are employed to evaluate them. To investigate the effectiveness of our proposed system employing either of two methods, we conduct human subject experiments on the motor selection problem and have revealed the following implications: (i) the proposed system based on the two fixed evaluation criteria contributes to helping users to find better motors in terms of all the evaluation criteria, while (ii) the proposed system based on the selected evaluation criteria is more effective to help users to understand Pareto optimal solutions when more evaluation criteria need to be considered.
\end{abstract}

Keywords: Pareto optimal solution, multi-dimensional space, interactive assistant system.

\section{Introduction}

In various decision making in human society, we generally have to explore solutions by considering multiple criteria rather than a single criterion. To derive an optimal decision-making in such a multi-dimensional space, it is necessary to understand trade-off relationship among multiple criteria. Since these relationships become complicated as the number of the evaluation criteria increases, it becomes to be hard to understand all of their relationships. When finding rental apartment, for example, it is 
not so difficult to find a good apartment by considering only a room size and a rental cost. However, it becomes to be very hard to find a satisfied apartment by considering a distance from a station, a resistance to earthquakes, and newness/oldness in addition to a room size and a rental cost. This is because it is generally difficult for us to perfectly understand the complicated relationship among many evaluations as the number of the evaluation criteria increases.

For this issue, the multi-objective optimization approach such as NSGA-II (Elitist Non-Dominated Sorting Genetic Algorithm) [1] is useful because it can find Pareto optimal solutions in the multi-dimensional space through an evaluation of solutions by considering all evaluation criteria. As another approach, a visualization of multidimensional space [5] is also useful because it can show the relationship among solutions in the two-dimensional space converted from the multi-dimensional space. These approaches can compute good solutions or can visualize the relationship among solutions, they cannot directly support humans to understand the trade-off relationships among them.

To tackle this problem, this paper proposes the interactive system that can support humans to understand the trade-off relationship of Pareto optimal solutions in multidimensional space through an interaction between the system and a user. For this purpose, we focus on an influence of changing a number and a kind of evaluation criteria which should be considered by a user at one time, and propose the following two methods: (i) the two fixed evaluation criteria are employed to evaluate the solutions; and (ii) some evaluation criteria selected by a user (i.e., the number of the evaluation criteria is varied by a user according to his or her understanding) are employed to evaluate them. A human subject experiments on a motor selection problem for space exploration rovers as multi-dimensional space problem are conducted to investigate the effectiveness of the proposed system from the viewpoint of the degree of understanding of the human subjects.

This paper is organized as follows. Section 2 explains the non-dominated sorting which is one of the multi-objective optimization, and Section 3 describes the proposed system employing either of both methods. Section 4 explains the human subject experiments on a motor selection problem, and Section 5 shows experimental results. Finally, our conclusion is given in Section 6.

\section{How to Evaluate Solutions in Multi-dimensional Space}

\subsection{Pareto Front}

The trade-off relationship among solutions is often occurred when the number of evaluation criteria is more than one. Considering the situation of finding rental apartment, for example, a rental cost increases if a distance between an apartment and a station becomes short, while a rental cost decreases if such a distance becomes long. To evaluate these solutions in the trade-off relationship, the non-dominated sorting calculates an order of solutions by finding Pareto optimal solutions [2] (i.e., a solution group which is the most superior to other solution groups) in multi-dimensional space.

Fig. 1 shows how the non-dominated sorting works. In Fig. 1, the points A, B, C, and $\mathrm{D}$ are respectively represented as $(\mathrm{x} 1, \mathrm{y} 1),(\mathrm{x} 2, \mathrm{y} 2),(\mathrm{x} 2, \mathrm{y} 1)$, and $(\mathrm{x} 1, \mathrm{y} 2)$ with the 
condition where $\mathrm{x} 1>\mathrm{x} 2$ and $\mathrm{y} 1>\mathrm{y} 2$. In such relationship, the point $\mathrm{A}$ dominates the points $\mathrm{B}, \mathrm{C}$, and $\mathrm{D}$, the point $\mathrm{B}$ dominates the point $\mathrm{D}$, and the point $\mathrm{C}$ dominates the point D. Concretely, the solutions represented by red circles in Fig. 1 are not dominated by other solutions, and they are called Pareto optimal solutions and a set of them is defined as the Pareto front 1 . Then, the set of solutions which are not dominated by other solutions except for the solutions in the Pareto front 1 is defined as the Pareto front 2. The Pareto front 3, 4, .., $\mathrm{n}$ are defined by the same way. According to this definition, the solutions in a small Pareto front number are superior to those in a large Pareto front number.

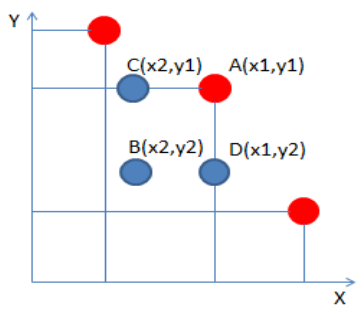

Fig. 1. Image of Pareto front solutions

\subsection{Average Pareto Front}

Using the concept of the Pareto front, this paper proposes average Pareto front, which is an average value of the Pareto front number in the multi-dimensional space. Concretely, the average Pareto front is calculated from all combination of two evaluation criteria. Table 1 shows an example of how the average Pareto front is calculated in the case of three evaluation criteria. For example, the average Pareto front in $x 2$ (1.67) is calculated by an average of three Pareto front numbers 2, 1, and 2, each of which is an average of the front numbers of 1 and $\mathrm{f} 2, \mathrm{f} 2$ and $\mathrm{f} 3$, and $\mathrm{f} 3$ and $\mathrm{f} 1$.

Table 1. Calculation of average Pareto front

\begin{tabular}{|c|c|c|c|c|c|c|}
\hline Solution & Value of $f 1$ & Value of $f 2$ & Value of $f 3$ & Evaluation criteria & Front & Average Front \\
\hline \multirow{3}{*}{$\mathrm{x} 1$} & \multirow{3}{*}{5} & \multirow{3}{*}{5} & \multirow{3}{*}{2} & $\mathrm{f} 1 \& \mathrm{f} 2$ & 1 & \multirow{3}{*}{$(1+1+1) / 3=1$} \\
\hline & & & & f2\&f3 & 1 & \\
\hline & & & & f3\&f1 & 1 & \\
\hline \multirow{3}{*}{$\mathrm{x} 2$} & \multirow{3}{*}{3} & \multirow{3}{*}{4} & \multirow{3}{*}{4} & $f 1 \& f 2$ & 2 & \multirow{3}{*}{$(2+1+2) / 3=1.67$} \\
\hline & & & & f2\&f3 & 1 & \\
\hline & & & & f3\&f1 & 2 & \\
\hline \multirow{3}{*}{$\times 3$} & \multirow{3}{*}{4} & \multirow{3}{*}{2} & \multirow{3}{*}{5} & f1\&f2 & 2 & \multirow{3}{*}{$(2+1+1) / 3=1.33$} \\
\hline & & & & f2\&f3 & 1 & \\
\hline & & & & f3\&f1 & 1 & \\
\hline
\end{tabular}

\section{Proposed Interactive System}

\subsection{Overview}

To promote users to understand the trade-off relationship of Pareto optimal solutions in the multi-dimensional space, we take an approach of (1) deleting bad solutions and 
(2) reducing/changing the number of evaluation criteria which should be considered by a user at one time. Such a solution deletion and evaluation criteria reduction enables user to easily understand the relationship of evaluation criteria. Regarding the evaluation criteria reduction, in particular, the proposed interactive system starts with two evaluation criteria among them. A sequence of the proposed interactive system shows as follows.

1. Among all solutions displayed in the system, a user selects one solution which he/she thinks it good.

2. The average Pareto front of the selected solution is calculated

3. A certain number of the solutions from the largest average value front (i.e., bad solutions) are deleted. Considering 1000 solutions, for example, the worst 100 solutions are deleted and the top 900 solutions are remained if a certain number of the deleted solutions in 100 . Note that if the selected solution is within the 100 bad solutions, the solutions from the worst one to the selected one are deleted instead of the worst 100 solutions

4. The remaining solutions are displayed to a user. In detail, these solutions are displayed from the viewpoint of the different two evaluation functions (described in Section 3.2.1) or they are displayed from the viewpoint of some evaluation functions which are added/deleted (described in Section 3.2.2).

5. Return to 1 until a user determines to find a desired solution.

\subsection{Reducing/Changing the Number of Evaluation Criteria}

In this paper, we propose the following two methods for reducing/changing the number of evaluation criteria: (1) two evaluation criteria method and (2) changing evaluation criteria method

Two Evaluation Criteria Method. This method decomposes many evaluation criteria that is more than one into a combination of the two evaluation criteria and displays solutions from the viewpoint of one of combinations that a user has not yet understood yet to promote a user to learn the trade-off relationship of such solutions. Since it is generally difficult for us to understand the multidimensional space with many evaluation criteria at the same time, a division of many evaluation criteria into two evaluation criteria makes it easy to understand the multidimensional space. Note that this method displays solutions to a user from the viewpoint of one of combinations of the two evaluation criteria, and some bad solutions are deleted from the viewpoint of the same combinations of the two evaluation criteria by calculating the average Pareto front value of the selected solution. This indicates that good solutions are displayed with a small number of evaluation criteria to facilitate understanding of the relationship among them, while bad solutions are deleted with the same evaluation criteria to reduce such solutions as fast as possible.

Figure 2 shows an example of the two evaluation criteria method with three evaluation criteria, $\mathrm{f} 1, \mathrm{f} 2$ and $\mathrm{f} 3$. The sequence of this method is shown as follows: (1) a user starts to select one solution (i.e., the red triangle dot) from the $2 \mathrm{D}$ area of $\mathrm{f} 1$ and $\mathrm{f} 2$; 
(2) the system calculates the Pareto front value from all combination of three criteria, i.e., f1-f2, f1-f3, and $\mathrm{f} 2-\mathrm{f} 3$, and selects one combination of two evaluation criteria that has the highest front value (bad value) as the combination that a user has not yet understood. In this case, the combination of $\mathrm{f1}-\mathrm{f} 3$ is selected because of the highest front value 3; (3) the system deletes the some bad solutions which are lower than the selected solution's one in the combination of $\mathrm{f1-f3}$; (4) the system displays the remaining solutions in the combination of $\mathrm{f} 1-\mathrm{f} 3$ and return to (1) until a user determines to find a desired solution.

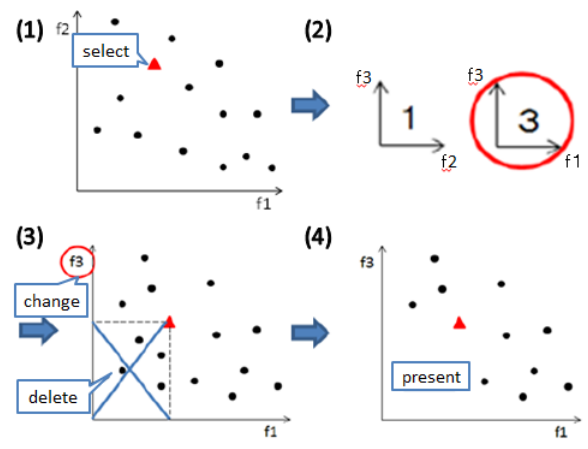

Fig. 2. Sequence of two evaluation criteria method

Changing Evaluation Criteria Method. This method enables a user to change (i.e., increase or decrease) the number of evaluation criteria which a user considers at the same time. A user can freely change it at the timing of the user to adjust his/her understanding of the multidimensional space. Since it is generally difficult to understand many trade-off relations from the beginning, it is reasonable to start by understanding of the simple relationship (i.e., the relationship between two evaluation criteria in this method) and then gradually increases the degree of difficulty of understanding by adding evaluation criterion one by one.

Figure 3 shows an example of the changing evaluation criteria method with three evaluation criteria, f1, f2 and $\mathrm{f} 3$. The sequence of this method is shown as follows: (1) a user starts to select one solution (i.e., the red triangle dot) from the 2D area of $\mathrm{f} 1$ and f2; (2) the system calculates the average Pareto front of the selected solution from the viewpoint of two evaluation criteria, i.e., f1-f2. In this case, the average Pareto front value of two evaluation criteria is calculated as 2 ; (3) the system deletes the some bad solutions which are lower than the selected solution's one from the viewpoint of the average Pareto front value; (4) the system displays the remaining solutions; (5) a user adds (or deletes) one evaluation criterion and select one solution. In this case, a user adds an evaluation criterion $\mathrm{f} 3$ and selects a new solution (i.e., the green square dot); (6) the system calculates the average Pareto front of the selected solution from the viewpoint of three evaluation criteria, i.e., f1-f2, f1-f3, and $\mathrm{f} 2-\mathrm{f} 3$, In this case, the average Pareto front value of all combination of three evaluation criteria is calculated as 2.33 ; (7) the system deletes the some bad solutions which are lower than the 


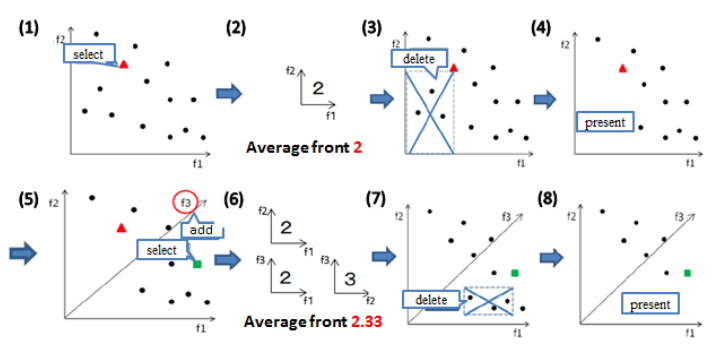

Fig. 3. Sequence of changing evaluation criteria method

selected solution's one from the viewpoint of the average Pareto front value; (8) the system displays the remaining solutions, and return to (5) until a user determines to find a desired solution.

\subsection{Comparing the Method by Using Simple Example}

We explain how each methods helps user with understanding the relationship by using the simple problem. The example is that there are three evaluate criteria and the number of the solution, (a)-(j), is ten. Table shows the relationship of its solutions, evaluate criteria and average front. Figure is the plot of the solution the combination of f1-f3 f1-f2andf2-f3.In this example, system presents the result of criteria is f1and f2at the beginning, user choice the solution of (d) and the number of deletion is two.

Table 2. Simple example of solution and average front

\begin{tabular}{|c|c|c|c|c|}
\hline Solution & Value of $\mathrm{f} 1$ & Value of $\mathrm{f} 2$ & Value of $\mathrm{f} 3$ & Average front \\
\hline ( a ) & 10 & 1 & 6 & 1.66 \\
\hline ( b ) & 9 & 3 & 4 & 2 \\
\hline ( c ) & 8 & 6 & 10 & 1 \\
\hline ( d ) & 7 & 9 & 2 & 2 \\
\hline ( e ) & 6 & 10 & 7 & 1.33 \\
\hline ( f ) & 5 & 5 & 1 & 2.66 \\
\hline ( g ) & 4 & 2 & 8 & 2.33 \\
\hline ( h ) & 3 & 7 & 3 & 2.33 \\
\hline ( i ) & 2 & 8 & 9 & 1.66 \\
\hline ( j ) & 1 & 4 & 5 & 3 \\
\hline
\end{tabular}

- Two evaluation criteria method. When user choice the solution of (d), system calculates the front value which the solution user selected belongs all combination in Figure 4. It shows (d)'s front value are 1, 2, and in the combination f1-f2 f2-f3 and f1$\mathrm{f3}$, and so system present the result of combination of $\mathrm{f1-f3}$. And then, in this combination, the system deletes the solution which has higher front value. In this case, the solutions of (h) and (j) are deleted. Next, in this evaluation criterion's combination f1-f3, assuming that the solution of (a) is chosen, the result of calculation, the new presentation's combination changes $\mathrm{f} 2 \mathrm{-} \mathrm{f} 3$ and deleted a certain number.

- Changing evaluation criteria method. When user choices the solution of (d), in the presented combination f1-f2, system deletes the solution which has high average 
front than selected solution a certain number. In this case, the solutions of $(\mathrm{g})$ and (j) are deleted. Next, assuming that user become considering the f3 criteria too, user selects the solution considered three criteria f1, f2 and f3. Then the solution of (c) is selected, and the deleted solutions which have higher average front than (c) become (f) and (h).

- Compared method. This method intends to compare the result of each method. The way of presentation is that all evaluation criteria are presented at the beginning and user can change the number of these. Specifically, when user choice the solution of (d), the system calculates the average front presented all evaluation criteria's combination, in this case $\mathrm{f} 1, \mathrm{f} 2$ and $\mathrm{f} 3$. And the solutions which has higher average front $((\mathrm{g})$ and $(\mathrm{j}))$ are deleted.

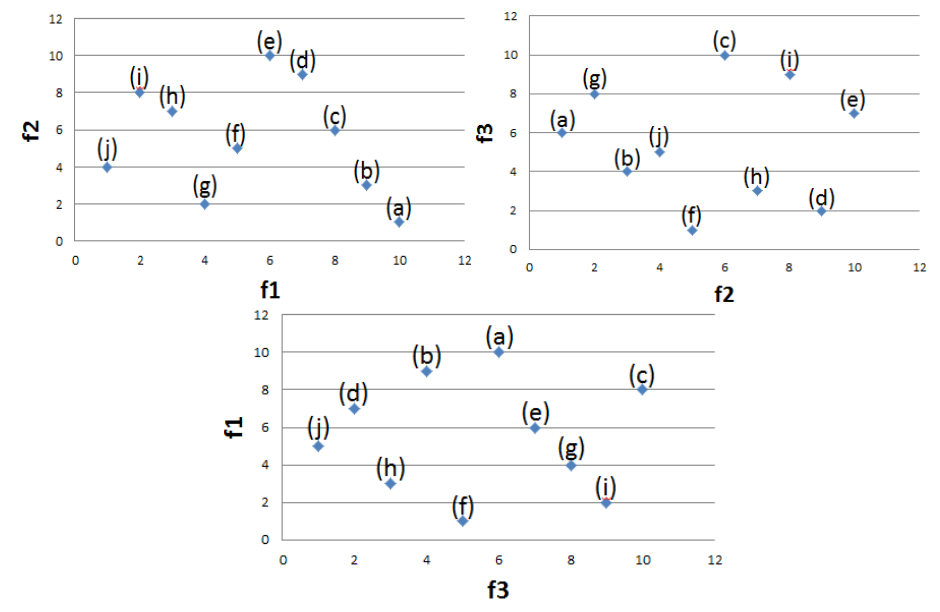

Fig. 4. Two-dimensional plot of the solution in simple problem

\section{$4 \quad$ Motor Selection Problem}

In the human subject experiments, we adopt the selection of motors for space exploration rover problem as Multi-Dimensional Space problem. The subjects refer to the data of the motor and select one motor from seven hundred motors. There are five evaluation criteria "Weight", "Length", "rpm", "Torque" and "Efficiency". In this case, the value of each evaluation criteria is important that the value of "Weight" is low, the value of "length" is low, the value of "rpm" is large, the value of "Torque" is high, and the value of the "Efficiency" is high.

\section{$5 \quad$ Human Subject Experiment}

To investigate the effect of the proposed system, we did human subject experiments. 


\subsection{Experimental Contents}

Subjects consist of nine people, six men and three women of 20s. They chose "most excellent performance" motor in the sequence of one experiment, and do this sequence total of five times. In this experiment, we set the problem special emphasis on the evaluation criteria of "Weight", "Torque" and "Length" in this case and inform user. This is because there are many cases when user searches for some solution, there is priority, so we assume such a situation. Flow of the experiment is as follows. First, we divided nine subjects into three groups, and there are three persons in one group. And we distribute the manual of experiment to subjects. The contents of manual shows that description of the contents of the experiment, the parameters used in the experiment, and how to operate the system. We have subjects read this and explain orally. Then subjects choose the best one motor and that selecting sequence was performed five times for a person.

\subsection{Evaluation Criteria}

We adopt evaluating the following three items in order to evaluate the degree of understanding of the multidimensional space; (1) average front of the solution chosen by subjects; (2) the time required for the final until subject selects the motor; (3) the similarity of the selected motor. If subject understand the relationship, they can chose the motor which has the low average front. Also, the time is short compared to one which does not understand presumably. Furthermore, if subject understand that, similar motor will be chosen.

\subsection{Experimental Result}

- Average front and Time of experiment. Figure5 shows that the average front of selected solution and time of each method. The horizontal axis shows each system, the vertical axis shows average front (left) and time (right). The difference of color between red and blue shows that the average front of all evaluation criteria and special emphasis on evaluation criteria. In two evaluation criteria method, the average front of special emphasis on evaluation criteria is the best of all. Also in changing the evaluation criteria method, the average front of all evaluation criteria and special emphasis on evaluation criteria is middle value of all methods.

- Transition of the selected motor. To examine whether the motor was finally selected by the subjects in each method, has changed how the number of times with each, we shows the distribution of motor in two-dimensional map by Multidimensional scaling method [4] (Figure 6). Multidimensional scaling method is that represented points in the space relationship of the object classification in lowdimensional distribution. Scatter diagram in this way shows that if the distance between the solution and other solutions that are plotted is short, nature of the solution are similar, if it is long, the nature of the solution is different. 
In the two evaluation criteria method, motor searching become in the narrow range gradually from a wide range. In the changing the evaluation criteria method, motor searching is always seen only in a narrow range.

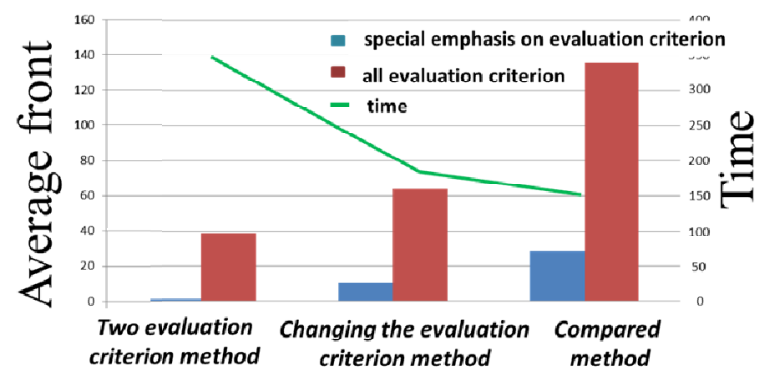

Fig. 5. Average front of selected solution and time of each method

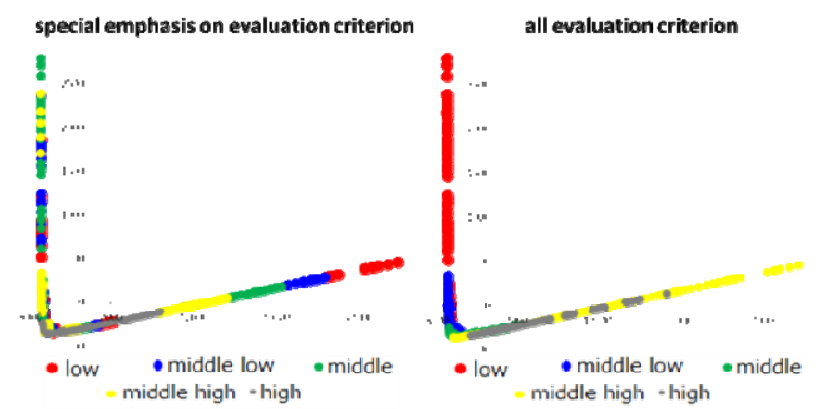

Fig. 6. The distribution of motor and average front by Multidimensional scaling method

\subsection{Discussion}

In this result, the two evaluation criteria method is that although searching time is long, subjects can choose the best motor in the proposed method and search as efficient transition, this method contribute to understand the multi-dimensional space. the changing the evaluation criteria method is that although it is possible to search a short time, slightly inferior motor has been selected compared with the two evaluation criteria method, this method contribute to understand local understanding of the solution space.

\section{Conclusion}

In this study, we proposed the interactive system that can help humans to understand the trade-off relationship of Pareto optimal solutions (e.g., good products from a certain aspect) in multi-dimensional space. For this purpose, the following two methods were proposed from the viewpoint of the number of evaluation criteria which should 
be considered by a user at one time: (i) the two fixed evaluation criteria are employed to evaluate the solutions; and (ii) some evaluation criteria selected by a user (i.e., the number of the evaluation criteria is varied by a user according to his or her understanding) are employed to evaluate them. To investigate the effectiveness of our proposed system employing either of two methods, we conducted human subject experiments on the motor selection problem for space exploration rovers as multidimensional space problem, and investigated the results from viewpoint of the averaged Pareto-front and the time for selecting one motor in order to evaluate the degree of understanding of the human subjects. An intensive analysis of the human subject experiments revealed the following implications: (i) the proposed system based on the two fixed evaluation criteria contributes to helping users to find better motors in terms of all the evaluation criteria. This means that users using this system can learn the trade-off relationship in the whole solution space effectively but it takes long time; and (ii) the proposed system based on the selected evaluation criteria is more effective to help users to understand Pareto optimal solutions when more evaluation criteria need to be considered. This means that users using this system can learn the trade-off relationship in the local solution space although it takes short time.

The following research must be done in the near future: (1) a generalization of the obtained implications by increasing the number of human subjects; and (2) a investigation of the effectiveness of the proposed system in other multipurpose design problem.

\section{References}

1. Deb, K.: Multiobjective Optimization using Evolutionary Algorithms. Wiley (2001)

2. Hiroyasu, T., Miki, M., Watanabe, S., Sakoda, T., Kamiura, J.: Evaluation of Genetic Algorithm for Objective Computation Methods. The Science and Engineering Review of Doshisha University 43(1), 41-52 (2002)

3. Kuroiwa, T.: Trade-off Analysis Method. Toshiba Special Reports, pp.48-50 (2005)

4. Taguchi, Y., Oono, Y., Yokoyama, K.: A New Eigenvector Technique for Multivariate Direct Gradient Analysis. Proceedings of the Institute of Statistical Mathematics 49(1), 133-153 (2001)

5. Yamashiro, D., Yoshikawa, T., Furuhashi, T.: Grasping the Effects of Genetic Operation and Improvement of Searching Ability through Visualizing Search Process for GA. Information Processing Society of Japan 48, 69-77 (2007) 